\title{
Emodialisi domiciliare oggi: i punti dii debolezza non risolti
}

\author{
Francesco Locatelli
}

\author{
Divisione di Nefrologia e Dialisi, Ospedale A. Manzoni, Lecco
}

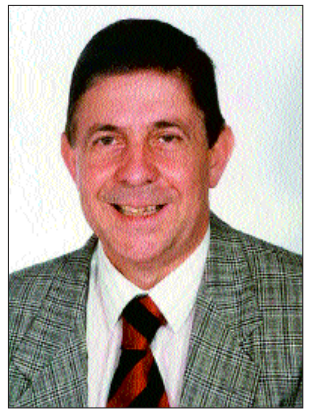

si domiciliare è
stata una grande
invenzione: ha
permesso di fron-
teggiare la crisi
dei posti tecnici
di dialisi, garan-
tendo la vita a
molti pazienti, che altrimenti sarebbero stati condannati a morire e quindi evitando angosce anche a noi nefrologi, altrimenti impossibilitati a dare una risposta alle vitali necessità dei pazienti. A un certo punto nel nostro Centro è successo il sorpasso: più pazienti a domicilio con l'emodialisi che al Centro. Successivamente, l'apertura dei Centri ad assistenza limitata ha fatto venir meno due delle principali motivazioni alla dialisi domiciliare. La più ovvia e importante era, come già detto, che l'emodialisi domiciliare era, per i pazienti, il solo modo per essere trattati e quindi continuare a vivere. La seconda motivazione, venuta meno con l'apertura dei Centri di assistenza limitata (CAL), era la lontananza dal Centro dialisi ospedaliero, con necessità per pazienti e familiari di lunghi trasferimenti, che spesso obbligavano i parenti a restare in ospedale per tut- ta la durata del trattamento emodialitico, aspettando il loro familiare. Ma quello che non si ricorda mai è lo stress del partner. Questo è stato, e penso sia ancora, il vero "tallone d'Achille" dell'emodialisi domiciliare. Non è possibile fare una dialisi "completamente da soli" ed è impensabile che una popolazione, mediamente sempre più anziana, possa dializzarsi "da sola" e autopungersi, indipendentemente dagli aspetti legislativi-normativi. Anche quando l'età media dei pazienti era 10 anni inferiore, l'autopuntura veniva effettuata da una minoranza dei pazienti e il familiare (anche egli ovviamente allora mediamente dieci anni più giovane!) doveva assumersi la responsabilità, il gravoso compito e lo stress di mettere le due agocannule, 3 volte alla settimana, in un sistema venoso mediamente molto migliore di quello attuale, non fosse altro perché i pazienti erano più giovani, la percentuale di diabete e arteriosclerosi più bassa e il sistema venoso meno sfruttato da una terapia conservativa che era ovviamente mediamente più corta. Ecco il punto: noi, anche con l'attuale tecnologia, e la teledialisi abbiamo bisogno di un partner che si assuma l'impegno a essere sempre disponibile e "in servizio" 3 volte alla settimana per tutto l'anno. Certo, potremmo dargli delle "pause" come abbiamo fatto in passato, ma egli si sentirà colpevolizzato da questi periodi di "liberazione" che si dovesse prendere. La tecnologia forse ridurrà lo stress dell'ipotensione severa, la teledialisi aiuterà molto a non far sentire partner e paziente soli, ma se la dialisi la vogliono fare di notte, chi dorme? Per la dialisi corta diurna-serale certo ci vuole la macchina-lavatrice ("one button machine") per cui basta schiacciare il bottone per partire e schiacciare ancora il bottone alla fine per finire, risciacquare e sterilizzare, con possibilità di riutilizzo per 15-30 giorni e sostituzione poi completa del dispositivo da parte dell'assistenza, in modo che pazienti e familiari non perdano altro tempo, se non quello dedicato strettamente al trattamento depurativo. Ma anche qui il problema è la puntura dell'accesso vascolare. Certamente i cateteri centrali non sono la soluzione, ancora gravati come sono da gravissimi rischi trombotici e infettivi. I nuovi dispositivi non danno ancora garanzie (oltre a essere estremamente costosi), per cui, per un'ampia diffusione di questo programma, non basta la "one button machine", ma ci vuole anche la soluzione sicura del problema dell'accesso vascolare. 
Una volta superata questa grave difficoltà, è più facile immaginare un più ampio sviluppo per questo tipo di dialisi ad alta riabilitazione sociale e clinica, anche per la flessibilità che l'orario corto può garantire, meglio conciliando quindi le esigenze lavorative e sociali del paziente e del partner. Ma non illudiamoci, avremo sempre di più una dialisi geriatrica! $\mathrm{Ci}$ troveremo sempre di più a fare dialisi domiciliari, scoprendo amaramente che il "domicilio" dei nostri pazienti sarà in realtà una "residenza per anziani"! Dopo queste amare considerazioni vorrei chiudere con una nota di ottimismo: mi auguro che in futuro la dialisi geriatrica sia la conseguenza di un programma di trapianto così sviluppato da aver selezionato pesantemente in negativo la popolazione rimasta in dialisi!

nefrologia@ospedale.lecco.it 